\section{Parâmetros para a programação de procedimentos da linha de cuidado do câncer do colo do útero no Brasil}

\author{
Parameters for programming line of care \\ procedures for cervical cancer in Brazil
}

\section{Parámetros para la programación de procedimientos en línea con el cuidado del cáncer de cuello uterino en Brasil}

Caroline Madalena Ribeiro 1 Maria Beatriz Kneipp Dias 1 Maria Asunción Sole Pla 1 Flávia Miranda Correa 1 Fábio Bastos Russomano 2 Jeane Glaucia Tomazelli 1

doi: 10.1590/0102-311X00183118

\section{Resumo}

Estimaram-se parâmetros para planejamento e programação da oferta de procedimentos para rastreamento, investigação diagnóstica e tratamento de lesões precursoras do câncer do colo do útero e, com base nessas estimativas, avaliou-se a adequação da produção nacional dos procedimentos realizados pelo Sistema Único de Saúde (SUS) em 2017. As estimativas foram calculadas utilizando como referencial as condutas preconizadas nas diretrizes nacionais para o rastreamento. Os dados referentes aos exames de rastreamento foram obtidos no Sistema de Informação do Câncer do Colo do Útero, e os dados de seguimento, em prontuários médicos de uma unidade de referência em patologia cervical. A produção nacional dos procedimentos foi obtida a partir de dados dos Sistemas de Informações Ambulatoriais e Hospitalares do SUS. Para cada cem mil mulheres na faixa etária alvo do rastreamento (25-64 anos), estimou-se a necessidade anual de 44.134 exames citopatológicos, 1.886 colposcopias, 275 biópsias, 236 excisões tipo 1 e 2 ambulatoriais, 236 excisões tipo 2 e 3 hospitalares e 39 encaminhamentos para alta complexidade para realização de cirurgia, quimioterapia e/ou radioterapia. Aplicando-se os parâmetros estimados ao número de mulheres rastreadas no Brasil em 2017, identificou-se déficit de todos os procedimentos para o seguimento adequado das mulheres com alterações, variando de 7\% nas colposcopias a 74\% nas excisões tipo 3. Os resultados apontam necessidade de ampliar e qualificar a oferta de procedimentos da linha de cuidado do câncer do colo do útero. Os parâmetros estimados poderão subsidiar gestores na programação e implementação de programas de rastreamento organizado.

Programas de Rastreamento; Neoplasias do Colo de Útero; Programação;

Planejamento em Saúde; Sistema Único de Saúde

\author{
Correspondência \\ C. M. Ribeiro \\ Instituto Nacional de Câncer José Alencar Gomes da Silva. \\ Rua Marquês de Pombal 125, 7o andar, Rio de Janeiro, RJ \\ 20230-240, Brasil. \\ cribeiro@inca.gov.br \\ 1 Instituto Nacional de Câncer José Alencar Gomes da Silva, Rio \\ de Janeiro, Brasil. \\ 2 Instituto Nacional de Saúde da Mulher, da Criança e do \\ Adolescente Fernandes Figueira, Fundação Oswaldo Cruz, Rio \\ de Janeiro, Brasil.
}




\section{Introdução}

O rastreamento do câncer do colo do útero por meio do exame citopatológico é uma estratégia de saúde pública capaz de reduzir a incidência e a mortalidade pela doença ${ }^{1}$. O impacto dessa ação pode ser comprovado pelas experiências bem-sucedidas no controle da doença 2 na Austrália, no Reino Unido, na Nova Zelândia e em países nórdicos.

No Brasil, diversas inciativas governamentais de incentivo ao controle do câncer do colo do útero têm ocorrido desde a década de $1980^{3}$. No entanto, o país ainda não conta com um programa organizado e apresenta taxas de incidência e mortalidade elevadas em comparação à dos países de alta renda 4 .

O Ministério da Saúde recomenda que mulheres entre 25 a 64 anos de idade realizem um exame a cada três anos após dois exames anuais consecutivos normais 5 . Apesar de estimar-se que $75 \%$ dos exames realizados no Sistema Único de Saúde (SUS) estejam dentro dessa faixa etária, há dificuldade no seguimento da periodicidade recomendada $6,7,8$.

Apesar da estimativa da Pesquisa Nacional de Saúde (PNS) de que 78\% das mulheres brasileiras tenham realizado exame preventivo nos três anos anteriores à pesquisa 9 , estimativas de cobertura do rastreamento no SUS calculadas pelos dados registrados nos sistemas de informação do SUS indicam níveis de cobertura inferiores a 50\% 10,11 .

A estabilidade das taxas de mortalidade do país e a queda apenas nas capitais 12 sinalizam que, apesar dos esforços de oferecer o rastreamento, não se tem alcançado a suficiência de oferta de procedimentos de todas as etapas da linha de cuidado para cobertura e continuidade do cuidado de toda população.

O planejamento e monitoramento são etapas essenciais para o sucesso de um programa de rastreamento 13. A existência de parâmetros de programação é de grande importância nesse processo, e sua elaboração constitui um desafio aos gestores da área da saúde.

Historicamente os parâmetros assistenciais do SUS tiveram caráter normativo, baseando-se em séries históricas de produção, sem considerar as evidências científicas para o cálculo das necessidades de saúde da população. Em 2015, foram lançados novos critérios técnicos que visam a fornecer referenciais para o planejamento e programação de ações e serviços de saúde no SUS, rompendo com a lógica anterior 14.

Embora o Brasil possua diretrizes clínicas 5 que definem os protocolos assistenciais para o rastreamento, não há parâmetros estabelecidos para a programação de procedimentos da linha de cuidado do câncer do colo do útero. Assim, o monitoramento, na maior parte dos casos, fica restrito às ações de rastreamento, sem englobar as ações de seguimento que incluem a investigação diagnóstica e o tratamento de lesões precursoras 15,16.

O objetivo deste estudo foi estimar parâmetros para o planejamento e programação da oferta de procedimentos para o rastreamento, investigação diagnóstica e tratamento de lesões precursoras do câncer do colo do útero, bem como avaliar a necessidade de sua adequação para um rastreamento populacional no Brasil.

\section{Métodos}

Os resultados de exames citopatológicos e histopatológicos no SUS são registrados no Sistema de Informação do Câncer do Colo do Útero (SISCOLO) 17 e no Sistema de Informação do Câncer (SISCAN), que vem gradualmente substituindo o primeiro desde 2013 18. No SISCOLO, não há identificação única das mulheres, não sendo possível avaliar sua trajetória no sistema de saúde de forma simples, mas apenas por meio de técnicas de relacionamento probabilístico.

Considerando as limitações das informações disponíveis e a necessidade de estabelecer parâmetros para o planejamento dos procedimentos da linha de cuidado do câncer do colo do útero baseado nas diretrizes nacionais para o rastreamento 5 , foram utilizados métodos e fontes de dados diferenciados, realizando-se este estudo em cinco etapas descritas a seguir. 


\section{Etapa 1 - análise da distribuição dos exames citopatológicos no Brasil}

Na primeira etapa, foi realizada uma análise descritiva para estimar a proporção de exames com alterações na população rastreada para câncer do colo do útero no SUS. Utilizou-se a distribuição dos resultados de exames citopatológicos do colo do útero, realizados no Brasil e registrados no SISCOLO, no período de 2011-2013, em mulheres na faixa etária alvo (25-64 anos).

Considerando que a qualidade dos laboratórios responsáveis pela realização do exame citopatológico influencia a distribuição dos resultados 19 , foram utilizados os dados dos exames realizados em laboratórios cujos indicadores de qualidade de citopatologia estavam dentro dos parâmetros estabelecidos pelo Ministério da Saúde para o credenciamento de laboratórios 20.

Embora a escala de produção de exames não seja obrigatória para o credenciamento, esse fator é importante para a garantia da qualidade, uma vez que confere a expertise necessária aos profissionais responsáveis pelos laudos dos exames 21 . Sendo assim, calculou-se a distribuição interquartílica da produção dos laboratórios previamente selecionados, mantendo apenas aqueles cujo volume de produção anual foi superior ao primeiro quartil.

Foram excluídos os laboratórios com índice de positividade superior a 10\%, considerado alto e frequentemente característico dos laboratórios de unidades de referência para investigação diagnóstica e tratamento de mulheres com alterações no rastreamento 19.

Foi calculada a proporção média dos diagnósticos citopatológicos, emitidos pelos laboratórios selecionados, com respectivos intervalos de 95\% de confiança (IC95\%). Também foram calculados a mediana e o intervalo interquartílico.

\section{Etapa 2 - análise dos procedimentos necessários para o seguimento das mulheres encaminhadas à colposcopia}

$\mathrm{Na}$ segunda etapa, foi realizado um estudo retrospectivo para estimar a necessidade de procedimentos para o seguimento de mulheres que tiveram alterações identificadas no exame citopatológico de rastreamento. Os dados foram extraídos dos registros eletrônicos de atendimentos realizados em um ambulatório de patologia cervical de referência no Estado do Rio de Janeiro. Quando necessário, para complementar dados faltantes, utilizaram-se os prontuários médicos.

O critério para seleção da unidade baseou-se na adoção rigorosa das condutas clínicas estabelecidas pelo Ministério da Saúde 5 e na disponibilidade de informações organizadas eletronicamente. Foram incluídas no estudo mulheres entre 25 e 64 anos de idade, atendidas entre julho de 2011 e dezembro de 2013. Foram excluídas mulheres gestantes e conhecidamente infectadas pelo HIV, por demandarem condutas clínicas diferenciadas 5 , além das mulheres com neoplasia intraepitelial vaginal (VAIN). Os dados foram agrupados segundo diagnóstico original, e foram calculadas as frequências absolutas e relativas, com respectivos intervalos de confiança, dos procedimentos realizados no seguimento.

\section{Etapa 3 - Estimativa da proporção de persistência de lesões de baixo de grau}

Segundo as diretrizes brasileiras 5 , mulheres que apresentam resultado citopatológico de lesão intraepitelial de baixo grau (LSIL) ou atipias escamosas de significado indeterminado possivelmente não neoplásicas (ASC-US) devem repetir o exame em 6 ou 12 meses, na dependência da idade. Quando há persistência dessas alterações, os casos devem ser encaminhados às unidades de referência para investigação colposcópica 5. Assim, não seria possível a partir dos dados da unidade de referência identificar a proporção de lesões persistentes, sendo necessária uma terceira etapa do estudo, na qual foram utilizados dados identificados do Estado do Rio de Janeiro, registrados no SISCOLO.

Foi criada uma base apenas com mulheres que tiveram diagnóstico citológico de LSIL ou ASC-US em 2011. Quando a mulher apresentava mais de um exame no ano, foi mantido o mais antigo. Posteriormente foi realizado relacionamento probabilístico dessa base com os exames do SISCOLO realizados em 2010, para excluir as mulheres que já haviam tido resultados alterados anteriormente, formando uma base de referência com as mulheres que tiveram o primeiro exame alterado (ASC-US ou LSIL) em 2011. A seguir foi realizado novo relacionamento entre a base de referência e as bases 
de 2011 e 2012 do SISCOLO, para identificar no seguimento o resultado do segundo exame e obter a proporção de LSIL ou ASC-US persistente. O período de seguimento de um ano foi definido com base nas diretrizes brasileiras 5 .

O relacionamento probabilístico seguiu as orientações apresentadas no manual do programa utilizado, sendo realizada padronização dos dados, definição de blocos lógicos para comparação (blocagem) e construção de escores para os pares formados (pareamento). Utilizaram-se como chaves de blocagem as variáveis nome, nome da mãe e data de nascimento, além de variáveis que transformam o primeiro e último nome da mulher e da mãe em código fonético soundex, minimizando o problema dos erros de digitação. Os parâmetros para comparação foram aqueles propostos no manual do programa utilizado para o relacionamento 22 . Foram analisados somente pares com escores positivos.

\section{Etapa 4 - cálculo dos parâmetros de necessidade}

A partir dos dados levantados nas etapas anteriores, estimou-se a necessidade de procedimentos para rastrear e seguir as mulheres na faixa etária alvo para o câncer do colo do útero.

A Figura 1 apresenta um resumo do fluxo de encaminhamento baseado nas diretrizes clínicas para o rastreamento do câncer do colo do útero, identificando as fontes utilizadas neste estudo para obter as informações necessárias à elaboração dos parâmetros propostos.

O cálculo dos parâmetros de programação, ou seja, da necessidade anual de procedimentos da linha de cuidado do câncer do colo do útero para um programa de rastreamento organizado, projetados para um cenário de cobertura de $100 \%$ da população alvo, foi realizado da seguinte forma:

(i) Necessidade de exames citopatológicos de rastreamento e repetição: somou-se (a) um terço da população feminina de 25-64 anos, considerando a periodicidade trienal do exame de rastreamento; (b) o número de exames a serem realizados no intervalo de um ano, obtido pela identificação de exames de primeira vez registrados no SISCOLO, uma vez que a periodicidade trienal do exame só é indicada após a realização de dois exames anuais consecutivos normais; (c) o número de exames insatisfatórios ou rejeitados (que demandam repetição imediata); e (d) o número de exames com resultado ASC-US ou lesão intraepitelial de baixo grau (que demandam repetição em 6 ou 12 meses).

(ii) Necessidade de exames citopatológicos para investigação diagnóstica: aplicou-se a proporção de exames citopatológicos para avaliação de canal endocervical e/ou revisão de lâmina realizados na unidade de referência desse estudo à proporção esperada de mulheres que seriam encaminhadas a uma unidade de referência (ou seja, que realizaram colposcopia para investigação diagnóstica).

(iii) Necessidade de exames citopatológicos para seguimento após resultado de biópsia normal/benigno: aplicou-se a proporção de exames para seguimento dos casos com resultado de biópsia normal/ benigno à proporção esperada de mulheres que seriam encaminhadas a uma unidade de referência. (iv) Necessidade de exames citopatológicos para seguimento após tratamento de lesão precursora: aplicou-se a proporção de exames para seguimento de mulheres após excisão tipo 1, 2 ou 3 (calculada com base na necessidade de citologia entre 6 e 12 meses após o procedimento e anual até completar cinco anos de seguimento - portanto seis exames de seguimento) à proporção esperada de mulheres que seriam encaminhadas a uma unidade de referência.

(v) Necessidade de colposcopias para investigação diagnóstica: calculou-se a proporção de mulheres com alterações identificadas no rastreamento, cuja recomendação é realizar esse exame.

(vi) Necessidade de colposcopias para seguimento: calculou-se a proporção de colposcopias realizadas para seguimento de mulheres após diagnóstico normal/benigno ou tratamento de lesões precursoras (considerando seguimento colposcópico semestral por um ano nos casos de margens livres) na unidade de referência e aplicou-se à proporção de mulheres que seriam encaminhadas a uma unidade de referência.

(vii) Necessidade de biópsias: calculou-se a proporção de biópsias realizadas na unidade de referência e aplicou-se à proporção de mulheres que seriam encaminhadas a uma unidade de referência.

(viii) Necessidade de excisão tipo 1 e excisão tipo 2 ambulatorial: calculou-se a proporção de excisões tipo 1 e excisões tipo 2 realizadas em nível ambulatorial pela unidade de referência e aplicou-se à proporção de mulheres que seriam encaminhadas a uma unidade de referência.

(ix) Necessidade de excisões tipo 2 realizadas em nível hospitalar e excisões tipo 3 (conização): calculou-se a proporção de excisões tipo 2 realizadas em nível hospitalar e excisões tipo 3, pela unidade 


\section{Figura 1}

Fluxograma das condutas clínicas recomendadas para o rastreamento do câncer do colo do útero e fontes de informação utilizadas no estudo.

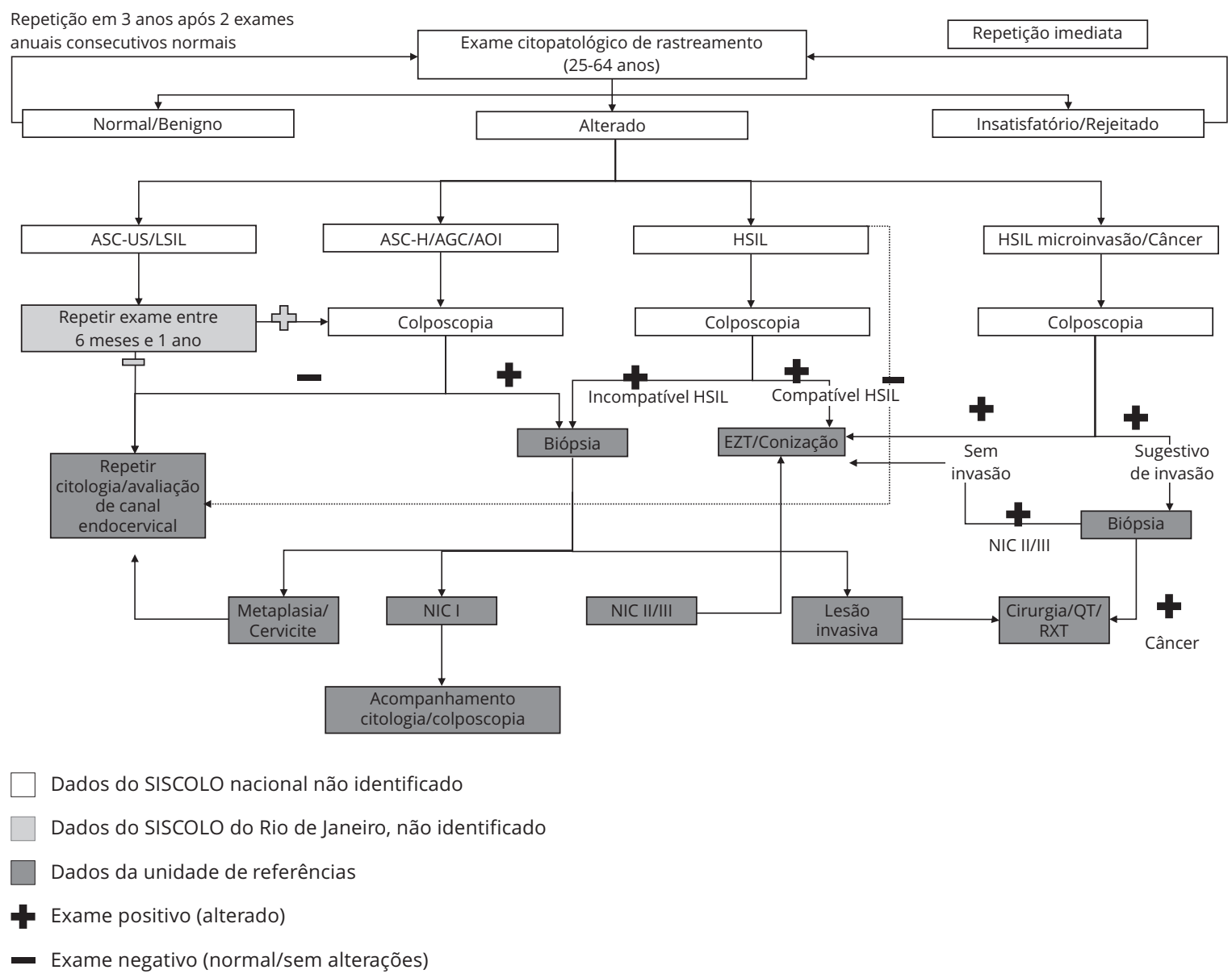

AGC: atipia glandular de significado indeterminado; AOI: células atípicas de origem indefinida; ASC-H: células escamosas atípicas de significado indeterminado não podendo excluir lesão intraepitelial de alto grau; ASC-US: células escamosas atípicas de significado indeterminado, provavelmente não neoplásicas; EZT: excisões; HSIL: lesão intraepitelial escamosa de alto grau; HSILM: lesão intraepitelial escamosa de alto grau que não pode excluir microinvasão; LSIL: lesão intraepitelial escamosa de baixo grau; QT: quimioterapia; RXT: radioterapia; SISCOLO: Sistema de Informação do Câncer do Colo do Útero.

de referência, e aplicou-se à proporção de mulheres que seriam encaminhadas a uma unidade de referência.

(x) Necessidade de encaminhamentos para alta complexidade (cirurgia, quimioterapia ou radioterapia): calculou-se a proporção de mulheres que tiveram diagnóstico confirmado de câncer na investigação diagnóstica ou após tratamento excisional e aplicou-se à proporção de mulheres que seriam encaminhadas a uma unidade de referência, excluídas aquelas submetidas a tratamento conservador do carcinoma microinvasor (estádio IA1).

\section{Etapa 5 - avaliação da oferta de procedimentos no SUS com base nos parâmetros estimados}

A população feminina considerada para estimativa de necessidades e avaliação da oferta no SUS foi a da projeção populacional estimada pelo Instituto Brasileiro de Geografia e Estatística (IBGE) para o ano de 2017, excluindo-se a proporção de mulheres cobertas por planos de saúde suplementar. A 
cobertura de saúde suplementar foi a divulgada pela Agência Nacional de Saúde Suplementar para a população feminina na faixa etária de 20-69 anos, em dezembro de 201723.

A produção de exames citopatológicos (códigos 0203010019 e 0203010086 ), biópsias de colo do útero (código 0201010666), colposcopias (código 0211040029) e excisões tipo 1 (código 0409060089) e tipo 2 (código 0409060305) ambulatoriais foi obtida no Sistema de Informações Ambulatoriais do SUS (SIA/SUS). A produção de excisões tipo 2 hospitalares e tipo 3 (código 0409060038) foi obtida no Sistema de Informações Hospitalares do SUS (SIH/SUS) para o ano de 2017. Foram aplicados filtros para o sexo feminino e idade entre 25 e 64 anos.

Foi calculada a necessidade de procedimentos para rastrear e dar seguimento a todas as mulheres usuárias do SUS em 2017. Considerando que atualmente o país ainda não dispõe de um programa de rastreamento organizado, foram calculadas também as necessidades de procedimentos para o seguimento das mulheres que foram de fato rastreadas pelo SUS em 2017. Considerou-se o número de exames citopatológicos registrados em 2017 como proxy do número de mulheres rastreadas.

O déficit ou excesso de procedimentos foi calculado pela diferença entre a necessidade estimada para o seguimento das mulheres rastreadas em 2017 e a produção informada. Os resultados foram apresentados em frequências absolutas e relativas.

O relacionamento entre as bases de dados foi realizado com base no programa Reclink 22, e a análise de dados, pelo programa $\mathrm{R}$ (http://www.r-project.org).

O estudo foi aprovado pelos comitês de ética do Instituto Nacional de Câncer José Alencar Gomes da Silva e do Instituto Nacional de Saúde da Mulher, da Criança e do Adolescente Fernandes Figueira, Fundação Oswaldo Cruz, sob o CAEE 61524816.9.0000.5274.

\section{Resultados}

No período de 2011 a 2013, foram registrados no país cerca de 23 milhões de exames citopatológicos no SISCOLO por 1.465 laboratórios. Desses, 225 (15,4\%) atenderam a todos critérios de qualidade estabelecidos pelo Ministério da Saúde. Para a estimativa de distribuição dos diagnósticos citopatológicos no rastreamento, foram utilizados dados de 141 laboratórios, com produção de exames superior ao primeiro quartil e positividade inferior a 10\%. Esses laboratórios registraram cerca de 3,3 milhões de exames no período.

A distribuição dos resultados dos exames é apresentada na Tabela 1.

Cerca de $4 \%$ dos exames realizados no período e classificados como satisfatórios tiveram resultados alterados. As alterações mais frequentes foram ASC-US $(38,4 \%)$, lesão de baixo grau $(28,7 \%)$ e lesão de alto grau (15,7\%).

A partir dos dados de 6.056 mulheres na faixa etária de 25-64 anos, com diagnóstico citopatológico de ASC-US ou LSIL, identificou-se persistência da lesão em 14,5\% (IC95\%: 13,3\%-15,7\%) das que tiveram diagnóstico de ASC-US e em 30,3\% (IC95\%: 28,5\%-32,2\%) das que tiveram diagnóstico original de LSIL.

Para estimar a necessidade de procedimentos para o seguimento de mulheres encaminhadas à colposcopia, foram analisados dados de 661 mulheres entre 25 e 64 anos atendidas na unidade de referência selecionada, das quais 534 (80\%) atenderam aos critérios de inclusão.

O diagnóstico mais frequente foi o de lesão intraepitelial de alto grau (49,1\%). A biópsia foi o procedimento realizado com maior frequência, tendo sido indicada para $33 \%$ das mulheres atendidas na unidade. A Tabela 2 apresenta o número absoluto de procedimentos realizados no seguimento das mulheres segundo grupos de diagnóstico original.

Estimou-se a necessidade de 177 exames citopatológicos para seguimento das mulheres que tiveram biópsias com resultado NIC I, o que corresponde a uma razão de 0,33 exames para cada mulher atendida na unidade de referência. Além disso, estimou-se a necessidade de 1.950 exames citopatológicos para o seguimento das mulheres submetidas a tratamentos excisionais, correspondendo a uma razão de 3,7 exames por mulher atendida na unidade de referência.

Ainda para o seguimento de mulheres tratadas por procedimentos excisionais, foi estimada a necessidade de 760 colposcopias, representando 1,42 exame por mulher atendida na unidade secundária. 


\section{Tabela 1}

Distribuição dos resultados dos exames citopatológicos de rastreamento do câncer do colo do útero em mulheres com idade entre 25 e 64 anos. Laboratórios selecionados do Sistema Único de Saúde. Brasil, 2011-2013.

\begin{tabular}{|c|c|c|c|c|c|}
\hline Resultado & $\mathbf{n}$ & $\%(I C 95 \%)$ & Mediana & $1^{\circ}$ quartil & $3^{\circ}$ quartil \\
\hline Normal & 3.161 .305 & $94,7(94,7-94,7)$ & 95,2 & 93,1 & 96,6 \\
\hline Insatisfatórios/Rejeitados & 38.821 & $1,2(1,2-1,2)$ & 0,7 & 0,2 & 1,8 \\
\hline Alterados * & 137.463 & $4,1(4,1-4,1)$ & 3,4 & 2,6 & 4,8 \\
\hline ASC-US & 52.817 & $38,4(38,2-38,7)$ & 35,6 & 24,9 & 43,5 \\
\hline ASC-H & 12.784 & $9,3(9,2-9,5)$ & 9,3 & 4,9 & 14,4 \\
\hline $\mathrm{AOI}$ & 533 & $0,4(0,4-0,4)$ & 0,1 & 0,0 & 0,5 \\
\hline AGC & 7.478 & $5,4(5,3-5,6)$ & 3,9 & 1,9 & 8,0 \\
\hline LSIL & 39.482 & $28,7(28,5-29,0)$ & 26,8 & 20,8 & 37,2 \\
\hline HSIL & 21.563 & $15,7(15,5-15,9)$ & 16,1 & 11,6 & 20,8 \\
\hline Câncer & 4.110 & $3,0(2,9-3,1)$ & 2,5 & 1 & 4,9 \\
\hline Total & 3.337 .589 & 100,0 & - & - & - \\
\hline
\end{tabular}

AGC: células glandulares atípicas de significado indeterminado; AOI: células atípicas de origem indefinida; ASC-H: células escamosas atípicas de significado indeterminado não podendo excluir lesão intraepitelial de alto grau; ASC-US: células escamosas atípicas de significado indeterminado, provavelmente não neoplásicas; HSIL: lesão intraepitelial escamosa de alto grau; IC95\%: intervalo de 95\% de confiança; LSIL: lesão intraepitelial escamosa de baixo grau.

Fonte: Sistema de Informação do Câncer do Colo do Útero (SISCOLO).

* As proporções de cada alteração foram calculadas em relação ao total de exames alterados. Embora pouco frequentes, alguns exames podem conter registro de mais de uma alteração, sendo considerado o resultado mais grave para adoção da conduta.

\section{Tabela 2}

Número de procedimentos da linha de cuidado do câncer do colo do útero realizados no seguimento de mulheres atendidas na unidade de referência do estudo, segundo alteração identificada no exame de rastreamento. Unidade de referência, 2011-2013.

\begin{tabular}{|c|c|c|c|c|c|c|}
\hline $\begin{array}{l}\text { Diagnóstico } \\
\text { original }\end{array}$ & $\mathbf{n}$ & $\begin{array}{c}\text { Citologia avaliação } \\
\text { de canal }\end{array}$ & Biópsia & $\begin{array}{c}\text { Excisão tipo I e II } \\
\text { ambulatorial }\end{array}$ & $\begin{array}{c}\text { Excisão tipo II } \\
\text { hospitalar e } \\
\text { tipo III }\end{array}$ & $\begin{array}{c}\text { Encaminhamento } \\
\text { para alta } \\
\text { complexidade }\end{array}$ \\
\hline ASC-US/LSIL & 127 & 30 & 59 & 9 & 17 & 2 \\
\hline ASCH/AGC/AOI & 128 & 53 & 54 & 27 & 36 & 7 \\
\hline HSIL & 262 & 35 & 55 & 118 & 103 & 16 \\
\hline HSILM/Câncer & 17 & 0 & 10 & 0 & 15 & 9 \\
\hline Total & 534 & 118 & 178 & 154 & 171 & 33 \\
\hline
\end{tabular}

AGC: atipia glandular de significado indeterminado; ASC-H: células escamosas atípicas de significado indeterminado não podendo excluir lesão intraepitelial de alto grau; ASC-US: células escamosas atípicas de significado indeterminado, provavelmente não neoplásicas; HSIL: lesão intraepitelial escamosa de alto grau; HSILM: lesão intraepitelial escamosa de alto grau que não pode excluir microinvasão; LSIL: lesão intraepitelial escamosa de baixo grau.

Com base nos resultados acima, foram estimados parâmetros para a programação de procedimentos da linha de cuidado do câncer do colo do útero, considerando um programa de rastreamento organizado com cobertura de $100 \%$ da população-alvo (Tabela 3).

A estimativa de necessidade de exames citopatológicos de rastreamento correspondeu a 39,8\% das mulheres na faixa etária alvo, considerando-se a periodicidade do exame (33,3\% das mulheres), a realização de exames anuais para mulheres que fizeram o exame pela primeira vez ( $6 \%$ das mulheres) e a necessidade de repetição de exames insatisfatórios e rejeitados dentre os exames de rastreamento realizados $(1,16 \% * 39,3 \%=0,47 \%)$. 


\section{Tabela 3}

Parâmetros estimados e necessidade de procedimentos para cada 100 mil mulheres/ano na faixa etária alvo (25-64 anos).

\begin{tabular}{|c|c|c|}
\hline Procedimento & Parâmetro utilizado & $\begin{array}{l}\text { Necessidade anual (por } 100.000 \\
\text { mulheres de } 25-64 \text { anos) }\end{array}$ \\
\hline Exames citopatológicos de rastreamento & $39,8 \%$ das mulheres na faixa etária alvo & 39.800 \\
\hline Exames citopatológicos de repetição & $2,8 \%$ das mulheres rastreadas & 1.100 \\
\hline $\begin{array}{l}\text { Exames citopatológicos para investigação de canal/revisão } \\
\text { de lâmina }\end{array}$ & $0,4 \%$ das mulheres rastreadas & 157 \\
\hline $\begin{array}{l}\text { Exames citopatológicos de seguimento após biópsia com } \\
\text { resultado normal/benigno }\end{array}$ & $0,7 \%$ das mulheres rastreadas & 275 \\
\hline $\begin{array}{l}\text { Exames citopatológicos de seguimento após tratamento } \\
\text { excisional }\end{array}$ & $7,2 \%$ das mulheres rastreadas & 2.830 \\
\hline Exames citopatológicos (total) & $44,1 \%$ das mulheres na faixa etária alvo & 44.134 \\
\hline Colposcopia para investigação diagnóstica & $2 \%$ das mulheres rastreadas & 786 \\
\hline Colposcopia para seguimento & $2,8 \%$ das mulheres rastreadas & 1.100 \\
\hline Total de colposcopia & $4,8 \%$ das mulheres rastreadas & 1.886 \\
\hline Biópsia & $0,7 \%$ das mulheres rastreadas & 275 \\
\hline Excisão tipo 1 e tipo 2 ambulatorial & $0,6 \%$ das mulheres rastreadas & 236 \\
\hline Excisão tipo 2 hospitalar e tipo 3 & $0,6 \%$ das mulheres rastreadas & 236 \\
\hline Encaminhamento para alta complexidade oncologia & $0,1 \%$ das mulheres rastreadas & 39 \\
\hline
\end{tabular}

Para estimar a necessidade dos procedimentos de investigação diagnóstica e seguimento, foi utilizado como base o número de mulheres rastreadas, ou seja 39,3\% da população alvo (33,3\% das mulheres, considerando a periodicidade trienal somada a $6 \%$ das mulheres, que fazem o exame pela primeira vez).

Estimou-se que 1,6\% (IC95\%: 1,59\%-1,61\%) das mulheres rastreadas têm diagnóstico citológico original de ASC-US e 1,20\% (IC95\%: 1,19\%-1,21\%) de LSIL. Sendo assim, 2,8\% delas (IC95\%: 2,78\%2,82\%) deveriam repetir o exame entre seis meses e um ano após o resultado.

Aproximadamente $22 \%$ das mulheres atendidas na unidade de referência realizaram exames citopatológicos para avaliação de canal endocervical ou revisão de lâmina. Considerando a estimativa de que $2 \%$ das mulheres rastreadas necessitam de investigação diagnóstica, obteve-se o parâmetro de $0,4 \%$ de necessidade de exames citopatológicos para avaliação de canal ou revisão de lâmina $(0,02 * 0,22)$.

Foram realizados 177 exames citopatológicos no seguimento de mulheres com resultado normal ou benigno na investigação diagnóstica, o que corresponde a uma razão de 0,33 exame para cada mulher atendida na unidade. Estimou-se, portanto, a necessidade de exames citopatológicos nessa modalidade de $0,7 \%\left(0,33^{*} 0,02\right)$ das mulheres rastreadas.

Já para o seguimento de mulheres tratadas por excisão tipo 1, 2 ou 3 foram necessários 1.950 exames citopatológicos, estimando-se a necessidade de 3,6 exames citopatológicos de seguimento por mulher atendida na unidade de referência, o que representa $7,2 \%\left(3,6^{*} 0,02\right)$ das mulheres rastreadas.

Assim, a partir das diferentes indicações de realização do exame, estimou-se a necessidade de exames citopatológicos correspondente a 44,13 exames para cada cem mulheres da população alvo.

A estimativa de necessidade de colposcopia para investigação diagnóstica foi de $2 \%$, considerando a proporção de diagnósticos alterados na distribuição nacional e as proporções de ASC-US e LSIL persistentes encontradas na segunda etapa do estudo $(1,4 \%+0,23 \%+0,36 \%)$. Mulheres tratadas por excisão tipo 1, 2 ou 3 ou com resultado de investigação diagnóstica normal/benigno são acompanhadas com exames citopatológicos e colposcópicos. A estimativa de necessidade de colposcopias de seguimento para esses casos foi de $2,8 \%(1,42 * 0,02)$ da população rastreada. 
A necessidade total de colposcopias foi, portanto, de 4,8\% da população rastreada $(0,02+0,028)$.

Após investigação colposcópica, 33,3\% das mulheres realizaram biópsia na unidade de referência. Portanto, a estimativa de necessidade de biópsia foi de $0,7 \%$ das mulheres rastreadas $\left(0,33^{*} 0,02 \%\right)$.

Dentre as mulheres acompanhadas na unidade de referência, 28,9\% realizaram Excisão tipo 1 ou 2 em ambiente ambulatorial e 32\% foram submetidas à excisão tipo 2 em ambiente hospitalar ou tipo 3 (conização), o que corresponderia, respectivamente, a 0,57\% e 0,63\% das mulheres rastreadas.

Foram encaminhadas pela unidade de referência para tratamento em estabelecimentos de alta complexidade em oncologia $6,2 \%$ das mulheres. A estimativa de casos que demandariam tratamento em alta complexidade foi de $0,1 \%$ das mulheres rastreadas.

Segundo dados do SIA/SUS, em 2017, foram realizados 6.843 .041 exames citopatológicos no Brasil em mulheres de 25-64 anos de idade. A estimativa de necessidade desses exames para rastreamento e seguimento de $100 \%$ das usuárias do SUS na faixa etária alvo seria de 17.624 .088 exames, com possível déficit de $61 \%$.

Como não há registro da indicação clínica dos exames (rastreamento, repetição ou seguimento), aplicou-se a proporção estimada de exames de rastreamento (91\%), obtendo-se uma estimativa de 6.227.167 mulheres rastreadas no ano de 2017. Assim, o cálculo da necessidade dos demais procedimentos para acompanhamento dessas mulheres indicou déficit de colposcopias (-7,3\%), biópsias $(-20,4 \%)$ e de procedimentos para o tratamento de lesões precursoras: excisões tipo 1 e 2 ambulatoriais (-74,8\%), excisão tipo 2 hospitalar e excisão tipo $3(-67,6 \%)$ (Tabela 4).

\section{Discussão}

Os parâmetros estimados neste estudo permitiram calcular a necessidade anual de exames de rastreamento e procedimentos para investigação diagnóstica e tratamento de lesões precursoras do câncer do colo do útero em um cenário de rastreamento organizado, com cobertura de $100 \%$ da população feminina de 25-64 anos de idade. O cálculo do número de exames necessários, a partir dos parâmetros levantados, utilizou uma base populacional de cem mil mulheres, que pode ser ajustada para realidades locais em municípios de menor porte.

Como o Brasil ainda não possui um programa de rastreamento organizado, foi realizada uma análise da adequação da oferta de procedimentos de continuidade do cuidado diretamente a partir dos exames citopatológicos registrados em 2017 nos sistemas de informação do SUS. Estimou-se déficit importante nos procedimentos de tratamento de lesões precursoras, tanto em nível ambulatorial (-74,8\%), quanto em nível hospitalar (-67,6\%). A produção de biópsias também foi inferior ao estimado como necessário $(-20,4 \%)$.

A utilização da média nacional de exames de primeira vez para estimar o parâmetro de necessidade anual de exames citopatológicos de rastreamento pode configurar uma limitação, considerando as variações regionais existentes no Brasil. Dados da PNS, realizada em 2013, estimaram que a pro-

\section{Tabela 4}

Diferença entre o número necessário e o realizado de procedimentos de investigação diagnóstica e tratamento de lesões precursoras do câncer do colo do útero no Sistema Único de Saúde (SUS). Brasil, 2017.

\begin{tabular}{lccc}
\hline Procedimentos & $\begin{array}{c}\text { Necessários * } \\
\text { (n) }\end{array}$ & $\begin{array}{c}\text { Realizados ** } \\
\text { (n) }\end{array}$ & $\begin{array}{c}\text { Déficit/Excesso } \\
\text { (\%) }\end{array}$ \\
\hline Colposcopia & 298.904 & 276.968 & $-7,3$ \\
Biópsia & 43.590 & 34.686 & $-20,4$ \\
Excisão tipo 1 e 2 ambulatorial & 37.363 & 9.398 & $-74,8$ \\
Excisão tipo 2 hospitalar e tipo 3 & 37.363 & 12.124 & $-67,6$
\end{tabular}

* Número de procedimentos estimados para atender 6.227.167 mulheres rastreadas pelo SUS em 2017;

** Número de procedimentos registrados no Sistema de Informações Ambulatoriais do SUS (SIA/SUS) no ano de 2017. 
porção de exames de primeira vez variou de 6,6\% na Região Sul a 12,3\% na Região Norte do país 9 . Dessa forma, a utilização da média nacional pode comprometer os resultados em análises locais, com a indicação de que sejam utilizados dados regionais para fins de planejamento local.

Configura-se como outra limitação a utilização de dados secundários não identificados para análise dos procedimentos realizados em 2017, não sendo possível afirmar que cada registro represente um procedimento realizado por cada mulher, o que pode subestimar os déficits encontrados. Por outro lado, a falha nos registros de procedimentos realizados pode superestimar os déficits encontrados.

Apesar de a estimativa de cobertura do citopatológico para rastreamento do câncer do colo do útero, realizada pela PNS em 2013, ser próxima ao recomendado pela Organização Mundial da Saúde (80\%), a ausência de registro real de cobertura do rastreamento para câncer do colo do útero no Brasil vem sendo apontada por diferentes estudos ao longo dos anos, que avaliam como baixa a cobertura no SUS 11,24,25,26.

O déficit de exames citopatológicos encontrado neste estudo foi superior ao encontrado na literatura para o Brasil, possivelmente por conta de os estudos disponíveis considerarem apenas a realização de um exame a cada três anos para cada mulher na faixa etária alvo, sem incluir os dois primeiros exames anuais, os exames de repetição no caso de diagnóstico inicial de ASC-US ou LSIL, além daqueles necessários para investigação diagnóstica e seguimento das mulheres.

Em estudo publicado com dados de 2015 24, foi encontrado déficit de cerca de $46 \%$ de exames citopatológicos. Apesar de terem sido incluídos exames de repetição e seguimento no primeiro ano de tratamento, não foram contabilizados os exames necessários para investigação diagnóstica, nem os do seguimento de cinco anos das mulheres tratadas para lesões precursoras. A diferença observada em relação à necessidade de procedimentos entre os estudos é consequência das diferenças metodológicas para o cálculo das estimativas. Neste estudo, a utilização de fontes de informações mais robustas e com identificação das mulheres permitiu melhor aproximação do cenário real de necessidades de exames.

Em 2017, foram realizados cerca de 6,5 milhões de exames no SUS, e a estimativa de necessidade para rastreamento e seguimento das mulheres com resultados alterados foi de 17 milhões de exames.

O número de biópsias realizadas em 2017 foi 20\% inferior ao estimado como necessário. Em conjunto com o déficit observado para procedimentos de tratamento de lesões precursoras $(67,6 \%$ a $74,8 \%$ ), os achados refletem as dificuldades de acesso a procedimentos de investigação diagnóstica e tratamento já retratadas em outros estudos realizados no país 27.

O maior déficit de tratamentos excisionais em relação às biópsias pode ser explicado pelas práticas da unidade analisada para a elaboração dos parâmetros, na qual o método "Ver e Tratar" é uma prática bem estabelecida e, portanto, mais largamente utilizada. Por outro lado, o método tem sido pouco adotado no país. Assim, se considerada a realização da biópsia em todos os casos tratados pelo método "Ver e Tratar", a estimativa de necessidade passaria a ser 0,9\% das mulheres rastreadas, e o déficit seria de $38 \%$.

Os dados registrados nos sistemas de informações do SUS indicam que há déficit na produção nacional de procedimentos relacionados à investigação diagnóstica e ao tratamento de lesões precursoras do câncer do colo do útero, o que compromete os resultados das ações ainda oportunísticas de rastreamento no país. Entretanto, vale ressaltar que a ausência ou a subnotificação de produção de procedimentos podem ter superestimado esse déficit, uma vez que o SIA/SUS é um sistema para o financiamento, e a alimentação de seus dados é por vezes limitada pelo teto financeiro de municípios e estados que, quando atingido, interrompe por vezes o repasse da informação 28 .

A persistência de alterações em mulheres que apresentaram resultados compatíveis com ASC-US e lesão de baixo grau tem sido estudada com diferentes intervalos e métodos de seguimento. Budal e colaboradores 29 avaliaram o seguimento de mulheres com esses resultados por um período de quatro meses a um ano, identificando persistência ou progressão em $27,9 \%$ dos casos registrados no programa de rastreamento norueguês. Em estudos de seguimento de mulheres com resultados ASC-US, observou-se uma variação de 8,6\% a 37,2\% na identificação de persistência ou aparecimento de lesão intraepitelial 30,31. No estudo, foi observada alteração em 20,9\% das mulheres que realizaram seguimento, sendo 14,5\% para aquelas cujo resultado inicial foi ASC-US e 30,3\% para lesão de baixo grau.

Destaca-se que a necessidade de procedimentos de seguimento para mulheres com diagnóstico LSIL e ASC-US baseou-se na recomendação vigente à época de que 100\% delas deveriam realizar 
um segundo exame seis meses após o primeiro resultado alterado. No entanto, os dados do estado do Rio de Janeiro evidenciaram que apenas $49,2 \%$ das mulheres tinham registro de segundo exame no período de um ano.

Por serem estimativas para a programação da oferta de procedimentos, os cálculos utilizaram como base $100 \%$ da população alvo, considerando-se o caráter universal do sistema de saúde brasileiro. No entanto, diferentes realidades locais, tanto em relação à cobertura de saúde suplementar como na disponibilidade de recursos podem levar gestores a utilizarem a população que mais se ajustar a sua realidade local, descontando a população coberta por saúde suplementar e/ou aplicando a meta de cobertura de rastreamento desejada.

$\mathrm{Na}$ organização e planejamento das ações de saúde, conhecer o quantitativo de procedimentos necessários contribui para avaliar a necessidade de contratualização ou não de novos serviços para atender a demanda estimada, no âmbito local ou regional, além de também evidenciar déficit ou sobreoferta de procedimentos ao avaliar as ações de controle do câncer do colo do útero.

Conclui-se que os parâmetros apresentados neste estudo podem nortear gestores do SUS na implantação, no monitoramento e na avaliação de programas de rastreamento. Como as estimativas se baseiam em condutas recomendadas pelo Ministério da Saúde frente ao resultado dos exames citopatológicos 5 , os parâmetros são válidos para o território nacional e podem ser ajustados conforme a distribuição local dos resultados, especialmente no que se refere aos exames de primeira vez, rejeitados e insatisfatórios.

\section{Colaboradores}

C. M. Ribeiro e M. B. K. Dias contribuíram na concepção, delineamento, coleta, análise e interpretação dos dados, redação e revisão crítica. M. A. S. Pla contribuiu na coleta, análise e interpretação dos dados e revisão crítica. F. M. Correa colaborou no delineamento do estudo e revisão crítica. F. B. Russomano colaborou na coleta de dados e revisão crítica do artigo. J. G. Tomazelli contribuiu na análise e interpretação dos dados, revisão crítica do conteúdo do artigo. Todos os autores aprovaram a versão final do artigo.

\section{Informações adicionais}

ORCID: Caroline Madalena Ribeiro (0000-00032690-5791); Maria Beatriz Kneipp Dias (00000002-5847-9830); Maria Asuncion Sole Pla (0000-0002-0278-0958); Flavia Miranda Correa (0000-0002-1105-2397); Fabio Bastos Russomano (0000-0001-7510-0485); Jeane Glaucia Tomazelli (0000-0002-2472-3444).

\section{Referências}

1. Peirson L, Fitzpatrick-Lewis D, Ciliska D, Warren R. Screening for cervical cancer: a systematic review and meta-analysis. Syst Rev 2013; 2:35.

2. Simonella L, Canfell K. The impact of a twoversus three-yearly cervical screening interval recommendation on cervical cancer incidence and mortality: an analysis of trends in Australia, New Zealand, and England. Cancer Causes Control 2013; 24:1727-36.

3. Instituto Nacional de Câncer. Viva mulher: câncer do colo do útero: informações técnicogerenciais e ações desenvolvidas. Rio de Janeiro: Instituto Nacional de Câncer; 2002.

4. Ferlay J, Soerjomataram I, Dikshit R, Eser S, Mathers CD. GLOBOCAN 2012: cancer incidence and mortality worldwide. Lyon: IARC CancerBase; 2013.

5. Instituto Nacional de Câncer José Alencar Gomes da Silva. Diretrizes brasileiras para o rastreamento do câncer do colo do útero. Rio de Janeiro: Instituto Nacional de Câncer José Alencar Gomes da Silva; 2016.

6. Instituto Nacional de Câncer José Alencar Gomes da Silva. Avaliação de indicadores das ações de detecção precoce dos cânceres do colo do útero e de mama - Brasil e regiões, 2013. Rio de Janeiro: Instituto Nacional do Câncer José Alencar Gomes da Silva; 2015.

7. Corrêa CSL, Lima AS, Leite ICG, Pereira LC, Nogueira MC, Duarte DAP, et al. Rastreamento do câncer do colo do útero em Minas Gerais: avaliação a partir de dados do Sistema de Informação do Câncer do Colo do Útero (SISCOLO). Cad Saúde Colet (Rio J.) 2017; 25:315-23. 
8. Instituto Nacional de Câncer José Alencar Gomes da Silva. Monitoramento das ações de controle dos cânceres do colo do útero e de mama. Informativo Detecção Precoce 2017; 8:1-8.

9. Theme Filha MM, Leal MC, Oliveira EFV, Esteves-Pereira AP, Gama SGN. Regional and social inequalities in the performance of Pap test and screening mammography and their correlation with lifestyle: Brazilian national health survey, 2013. Int J Equity Health 2016; 15:136.

10. Instituto Nacional de Câncer José Alencar Gomes da Silva. Monitoramento das ações de controle dos cânceres do colo do útero e de mama. Rio de Janeiro: Instituto Nacional de Câncer José Alencar Gomes da Silva; 2012. (Boletim, 8).

11. Costa RFA, Longatto-Filho A, Pinheiro C, Zeferino LC, Fregnani JH. Historical analysis of the Brazilian cervical cancer screening program from 2006 to 2013: a time for reflection. PLoS One 2015; 10:e0138945.

12. Girianelli VR, Gamarra CJ, Azevedo e Silva G. Disparities in cervical and breast cancer mortality in Brazil. Rev Saúde Pública 2014; 48:459-67.

13. Andermann A, Blancquaert I, Beauchamp S, Déry V. Revisiting Wilson and Jungner in the genomic age: a review of screening criteria over the past 40 years. Bull World Health Organ 2008; 86:317-9.

14. Ministério da Saúde. Portaria no 1.631, de 1o de outubro de 2015. Aprova critérios e parâmetros para o planejamento e programação de ações e serviços de saúde no âmbito do SUS. Diário Oficial da União 2015; 2 out.

15. Ministério da Saúde. Portaria no 399/GM, de 22 de fevereiro de 2006. Divulga o Pacto pela Saúde 2006 - Consolidação do SUS e aprova as Diretrizes Operacionais do Referido Pacto. Diário Oficial da União 2006; 23 fev.

16. Ministério da Saúde. Resolução no 5, de 19 de junho de 2013. Dispõe sobre as regras do processo de pactuação de Diretrizes, Objetivos, Metas e Indicadores para os anos de 2013 - 2015, com vistas ao fortalecimento do planejamento do Sistema Único de Saúde (SUS) e a implementação do Contrato Organizativo da Ação Pública da Saúde (COAP). Diário Oficial da União 2013; 20 jun.

17. Ministério da Saúde. Portaria no 287, de 24 de abril de 2006. Diário Oficial da União 2006; 25 abr.

18. Ministério da Saúde. Portaria no 3.394/GM de 30 de dezembro de 2013. Institui o Sistema de Informação de Câncer (SICAN) no âmbito do Sistema Único de Saúde (SUS). Diário Oficial da União 2013; 31 dez.

19. Coordenação de Prevenção e Vigilância, Instituto Nacional de Câncer José Alencar Gomes da Silva. Manual de gestão da qualidade para laboratório de citopatologia. Rio de Janeiro: Instituto Nacional de Câncer José Alencar Gomes da Silva; 2016.
20. Ministério da Saúde. Portaria no 1.504 , de 23 de julho de 2013. Institui a Qualificação Nacional em Citopatologia na prevenção do câncer do colo do útero (QualiCito), no âmbito da Rede de Atenção à Saúde das Pessoas com Doenças Crônicas. Diário Oficial da União 2013; 24 jul.

21. Miller AB, Nazeer S, Fonn S, Brandup-Lukanow A, Rehman R, Cronje H, et al. Report on consensus conference on cervical cancer screening and management. Int J Cancer 2000; 86:440-7.

22. Camargo Jr. KR, Coeli CM. Reclink: aplicativo para o relacionamento de bases de dados, implementando o método probabilistic record linkage. Cad Saúde Pública 2000; 16:439-47.

23. Agência Nacional de Saúde Suplementar. Taxa de cobertura de planos de saúde. http://www. ans.gov.br/anstabnet/cgi-bin/dh?dados/tab net_tx.def (acessado em 15/Jul/2018).

24. Ribeiro CM, Azevedo e Silva G. Avaliação da produção de procedimentos da linha de cuidado do câncer do colo do útero no Sistema Único de Saúde do Brasil em 2015. Epidemiol Serv Saúde 2018; 27:e20172124.

25. Dias MBK, Tomazelli JG, Assis M. Rastreamento do câncer de colo do útero no Brasil: análise de dados do Siscolo no período de 2002 a 2006. Epidemiol Serv Saúde 2010; 19:293306.

26. Nascimento GWC, Pereira CCA, Nascimento DIC, Lourenço GC, Machado CJ. Cervical cancer screening coverage in the state of Minas Gerais, Brazil between 2000-2010: a study using data from the Cervical Cancer Information System (SISCOLO). Cad Saúde Colet (Rio J.) 2015; 23:253-60.

27. Farias ACB, Barbieri AR. Seguimento do câncer de colo de útero: estudo da continuidade da assistência à paciente em uma região de saúde. Esc Anna Nery Rev Enferm 2016; 20:e20160096.

28. Pires M, Vieira R. Medidas preliminares de produção na saúde pública. Carta de Conjuntura 37. http://www.ipea.gov.br/portal/ima ges/stories/PDFs/conjuntura/171027_cc_37_ nt_1.pdf (acessado em 12/Set/2018).

29. Budal EB, Haugland HK, Skar R, Maehle BO, Bjørge T, Vintermyr OK. HPV DNA testing improves $\mathrm{CIN} 2+$ risk stratification and detection of CIN2+ in delayed triage of ASCUS and LSIL. A population-based follow-up study from Western Norway. Cancer Med 2014; 3:182-9.

30. Cheung AN, Szeto EF, Ng KM, Fong KW, Yeung AC, Tsun OK, et al. Atypical squamous cells of undetermined significance on cervical smears: follow-up study of an Asian screening population. Cancer 2004; 102:74-80.

31. ASCUS-LSIL Traige Study (ALTS) Group. Results of a randomized trial on the management of cytology interpretations of atypical squamous cells of undetermined significance. Am J Obstet Gynecol 2003; 188:1383-92. 


\section{Abstract}

The study estimated parameters for planning and programming the supply of procedures for screening, diagnostic workup, and treatment of precursor lesions of uterine cervical cancer. These estimates were used as the basis for assessing the adequacy of Brazil's production of procedures performed by the Brazilian Unified National Health System (SUS) in 2017. Estimates were calculated using as the reference the recommended management in the national screening guidelines. Data on screening tests were obtained from the Information System on Uterine Cervical Cancer and the follow-up data from patient charts in a referral center for cervical pathology. Brazil's production of procedures was obtained from data in the Outpatient and Hospital Information Systems of the SUS. For every one hundred thousand women in the target age bracket for screening (25 to 64 years) there was an estimated annual need for 44,134 cytopathology tests, 1,886 colposcopies, 275 biopsies, 236 type 1 and 2 outpatient exicision procedures, 236 type 2 and 3 hospital exicision procedures, and 39 high-complexity referrals for surgery, chemotherapy, and/or radiotherapy. Applying the estimated parameters to the number of women screened in Brazil in 2017, a deficit was identified in all the procedures for adequate follow-up of the women with altered test results, varying from $7 \%$ in colposcopies to $74 \%$ in type 3 excisions. The results point to the need to expand and upgrade the supply of line of care procedures for cervical cancer. The estimated parameters can support policymakers in programming and implementing organized screening programs.

Mass Screening; Uterine Cervical Neoplasms; Programming; Health Planning; Unified Health System

\section{Resumen}

Se estimaron parámetros para la planificación y programación de la oferta de procedimientos, con el fin de detectar, realizar investigación diagnóstica y tratar lesiones precursoras del cáncer de cuello uterino. En base a las mismas, se evaluó la adecuación del protocolo nacional de procedimientos realizados por el Sistema Único de Salud (SUS) en 2017. Las estimaciones se calcularon utilizando como marco de referencia los procedimientos recomendados en las directrices nacionales para la detección de este tipo de cáncer. Los datos referentes a los exámenes de detección se obtuvieron en el Sistema de Información del Cáncer de Cuello de Útero, y los datos de seguimiento en registros médicos de una unidad de referencia en patología cervical. La producción nacional de los procedimientos se obtuvo a partir de datos de los sistemas de información ambulatoria y hospitalaria del SUS. De cada cien mil mujeres, en la franja de edad objetivo de la detección (25 a 64 años), se estimó una necesidad anual de 44.134 exámenes citopatológicos, 1.886 colposcopias, 275 biopsias, 236 escisiones tipo 1 y 2 ambulatorias, 236 escisiones tipo 2 y 3 hospitalarias y 39 derivaciones hacia centros hospitalarios de alta complejidad para la realización de cirugías, quimioterapia y/o radioterapia. Aplicando los parámetros estimados al número de mujeres a quienes se les realizó el examen en Brasil durante 2017, se identificó un déficit de todos los procedimientos para un seguimiento adecuado de las mujeres con alteraciones, variando de un $7 \%$ en las colposcopias, a un $74 \%$ en las escisiones tipo 3. Los resultados apuntan la necesidad de ampliar $y$ dotar la oferta de procedimientos en la línea de cuidados del cáncer de cuello uterino. Los parámetros estimados podrán ayudar a los gestores en la programación e implementación de programas organizados de detección.

Tamizaje Masivo; Neoplasias del Cuello Uterino; Programación; Planificación en Salud; Sistema Único de Salud
Recebido em 20/Set/2018

Versão final reapresentada em 08/Jan/2019

Aprovado em 06/Fev/2019 